\title{
AGN/Starburst Connection
}

\author{
Eleonora Sani ${ }^{1}$
}

\author{
${ }^{1}$ INAF - Osservatorio Astrofisico di Arcetri \\ Corresponding author: sani@arcetri.astro.it
}

\begin{abstract}
Two main physical processes characterize the activity in the nuclear region of active galaxies: an intense star formation (starburst, SB) and an Active Galactic Nucleus (AGN). While the existence of a starburst-AGN connection is undisputed, still it is not clear which process dominates the energetic output in both local and high redshift Universe. Moreover there is no consensus on whether AGN fueling is synchronous with star formation or follows it during a post-starburst phase. Here I first review how to disentangle the relative SB-AGN contribution, then I focus on the physical and geometrical properties of the circumnuclear environment.
\end{abstract}

Keywords: AGN - starburst - merging - feedback - imaging - spectroscopy - mid-IR - X-rays.

\section{Introduction}

The issue of AGN - star formation (AGN-SF) connection in local and distant galaxies is possibly relevant for understanding several processes: from galaxy formation and evolution, and the star formation and metal enrichment history of the Universe, to the the origin of the extragalactic background at low and high energies and the origin of nuclear activity in galaxies. It is well known that SF traces the growth of a galaxy in terms of stellar mass, and that galaxies assemble their mass through SB episodes. For example, local ULIRGs (Ultraluminous Infrared Galaxies) and high-z SMGs (Submillimeter galaxies) harbor the large molecular-gas reservoirs that are necessary to switch on SB episodes (Tacconi et al. 2010, 2013). On the other hand, AGNs trace the growth of supermassive black holes (SMBHs, with masses $\mathrm{M}_{\mathrm{BH}}>10^{6} \mathrm{M}_{\odot}$ ) and quiescent SMBHs are believed to dwell in almost all galaxy bulges as AGN relics, i.e. the result of a past AGN activity (Soltan 1982, Marconi et al. 2004, Merloni \& Heinz 2008).

\subsection{Evidence of AGN-SF connection}

A direct link between bulge formation and the growth of the central SMBHs has been inferred from tight relations between $\mathrm{BH}$ mass $\mathrm{M}_{\mathrm{BH}}$ and the bulge structural parameters, such as velocity dispersion (Ferrarese \& Merrit 2000, Gebhardt et al. 2000, Gultekin et al. 2009), luminosity (Kormendy \& Richstone 1995, Marconi \& Hunt 2003, Sani et al.2011) and mass (Magorrian et al. 1998, MH03, Häring \& Rix 2004). Since a SB is a natural consequence of dissipative gaseous processes as- sociated with spheroid formation (e.g. Barnes \& Hernquist 1991) and AGN-SF connection dating back to the early Universe is implied by these results, a mergerdriven scenario is well suited to reproduce such scaling relations.

Recently, attention has been drawn to the coexistence of pseudobulges and BHs (Nowak et al. 2010, Sani et al. 2011). Indeed pseudobulges seem to follow their own relation with $\mathrm{BHs}$, hosting less massive $\mathrm{BHs}$ than classical bulges (Greene, Ho, \& Barth 2008, Hu 2009), or they are at least displaced from the scaling relations for classical bulges (Sani et al. 2011, Kormendy et al. 2011). This may indicate a dichotomy in the formation history of galaxies (major merger vs. secular evolution) and/or that activity in galaxy nuclei can be stochastically driven by local processes such as minor mergers, bar/disk instabilities.

In the following I concentrate the discussion on the major-merger scenario, its implications and observational evidences. The reader is anyhow advised about the importance of secular evolution processes, especially in low-luminosity, local Seyfert galaxies.

\subsection{The major-merger scenario (ULIRGs-QSO path)}

The merger between galaxies of approximately the same size is relevant for the cosmological evolution of SBs, quasars and early type galaxies (Sanders et al. 1988, Silk \& Rees 1998, Fabian 1999, Granato et al. 2001, Di Matteo et al. 2005, Hopkins et al. 2008, Menci et al. 2008). A schematic view of the main steps in the merging process is given in Fig. 1. 


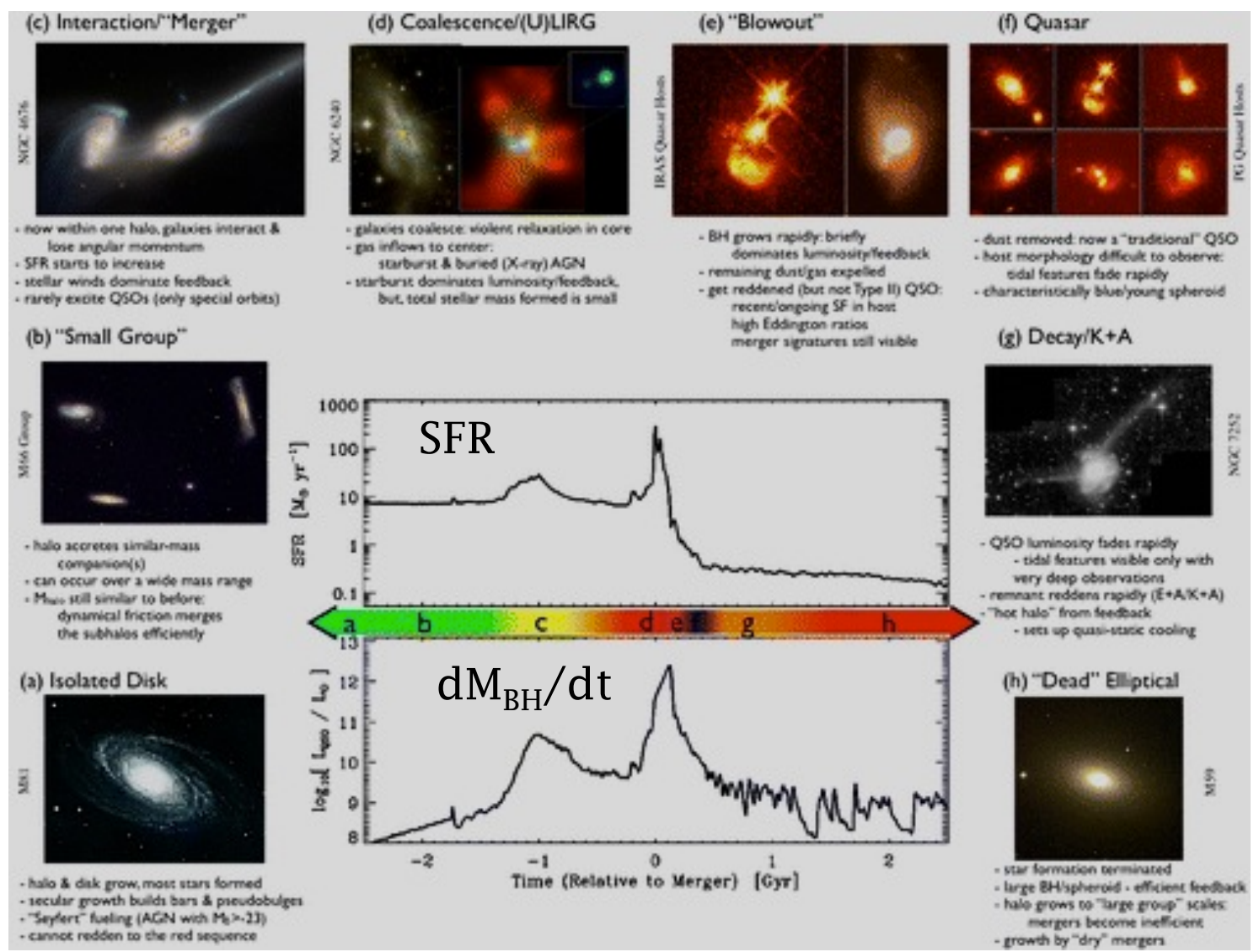

Figure 1: Major-merger scenario. Starting from the merger of two spiral galaxies (left side), the merger produces a violent SB and the onset of QSO activity (middle) and ends with the formation of a dead spheroid (right side). From Hopkins et al. 2008.

During first encounters, the mergers between gasrich galaxies (i.e. spirals) drag the gas which fuels both SF and AGN activity. Thus a violent SB occurs (ULIRG-phase) as well as heavily embedded SMBH growth (observed as obscured AGN). Then, the peak of accretion happens when the systems coalesce. At this point the quasar is cleaning the circumnuclear environment thanks to strong winds that blow out the gas, the AGN becomes X-ray and optically thin.

Finally, due to feedback processes both $\mathrm{BH}$ growth and further SF are quenched leaving a QSO relic in a red galaxy (passive evolution). Therefore, obscured QSO and feedback processes are key ingredients in the $\mathrm{BH}-$ host galaxy co-evolution. In the following, after a brief description of the AGN and SF emission, I review the AGN-SF observations for both single sources (interesting for their physics/structure) and large samples (to infer statistical properties).

\section{The AGN vs SF Emission}

An issue in AGN-SF studies is to correctly identify AGNs that represent only a small fraction of sources compared to the population detected at optical and IR wavelengths, and their properties can be elusive. Obscured AGNs can be elusive because their optical emission resembles that of normal galaxies and therefore they cannot be identified by the color-selection techniques used for QSOs, or because their spectra do not show any AGN signature. Their IR emission can be dominated by PAH features, typical of star-forming galaxies, instead of continuum emission from hot dust. Radio observations reveal the radio-loud population of obscured AGNs (e.g., radio galaxies), but this comprises only a small fraction, $10 \%$, of the total AGN population. A hard X-ray selection fails to detect the luminous narrow-line AGNs in the numbers predicted by some models of the cosmic X-ray background.

Polletta and collaborators (2007) show the differences in the spectral energy distributions (SEDs) of AGN and star forming galaxies. The average SEDs of the three classes show some clear differences. They become increasingly blue in the optical-near-IR $(\lambda<$ $1 \mu \mathrm{m}$ ), warmer in the IR (i.e. red at $\lambda \simeq 1-10 \mu \mathrm{m}$ ), and brighter in X-rays in the sequence $\mathrm{SF} \rightarrow \mathrm{AGN} 2 \rightarrow \mathrm{AGN} 1$. 
More in detail, most of the diagnostics developed till recently fail in identifying an AGN when it is intrinsically faint and/or deeply obscured. As an example of a possible case where the AGN is hardly detectable, I consider in Fig. 2 an intrinsically faint AGN, contributing only $20 \%$ to the bolometric source luminosity, and moreover its primary emission is absorbed by gas and dust. Only two spectral regions are useful for such an AGN detection: the hard X-rays (above $2 \mathrm{keV}$ ) and the near-mid infrared between 3 and $10 \mu \mathrm{m}$. Indeed in the near-IR and optical ranges the dust and gas extinction respectively avoid the AGN detection, and at radio wavelengths the $\mathrm{SB}$ emission peak dilutes AGN emission properties.

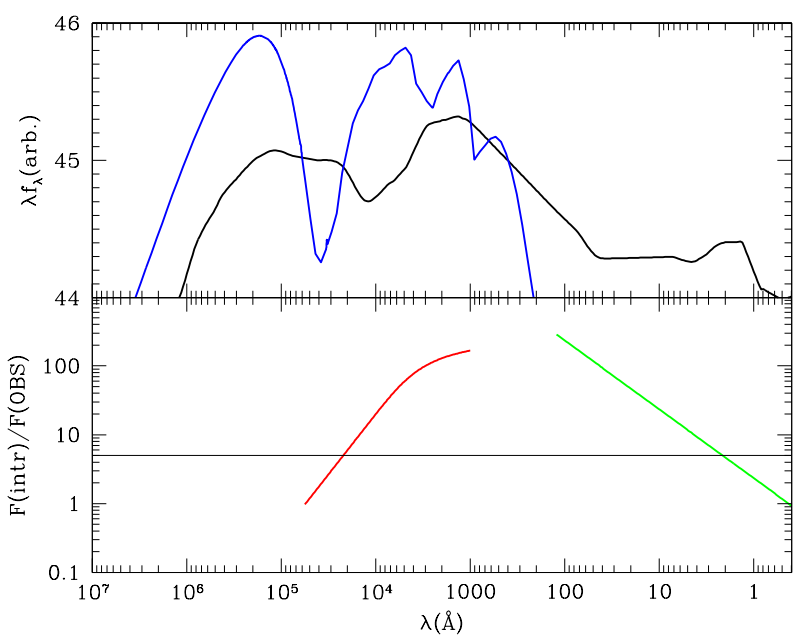

Figure 2: Top: simulated SEDs of a SB (blue) and an AGN (black) contributing to $80 \%$ and $20 \%$ respectively of the $\mathrm{L}_{b o l}$. Bottom: extinction of the AGN emission by gas (green) and dust (red), the horizontal black line indicates an absorption factor of 5. The AGN is undetectable in the range $3 \mu \mathrm{m}-2 \AA$ due to dust and gas extinction, and at $\lambda \geq 10 \mu \mathrm{m}$ for the SB dilution. Only the hardest X-rays and 3-10 $\mu \mathrm{m}$ observations reveal the AGN.

It is thus clear that the diagnostic performed in the $\mathrm{X}$-ray and IR bands can highlight the main AGN-SF differences and represent the wavelength rages where we do have the most detailed information respectively.

\section{X-Ray and IR Screenings: Imaging and Spectroscopy}

Here I briefly describe recent results obtained thanks to X-ray - IR imaging and spectroscopy in two puzzling sources. The final goal is to constrain the physics of ongoing processes and the structure of the nuclear region.

\subsection{The case of NGC 6240}

Thanks to imaging techniques we can simultaneously observe the AGN and SF activity in the nuclei of local galaxies (e.g. NGC 1068, NGC 6240, Mrk 231, Arp 299, Circinus...). The angular resolution represents the only limit for this analysis. NGC 6240 is a well known double-merging system $107 \mathrm{Mpc}$ far away. Here the Xray imaging and spectroscopy performed with Chandra (Komossa et al. 2003) revealed a Compton Thick double AGN, that was confirmed at all wavelengths, plus a SB covering a projected $\sim 2 \mathrm{kpc}$ scale (Risaliti et al. 2006, Medling et al. 2011, Feruglio et al. 2013).

HST and Spitzer images (Bush et al. 2008) show how the thermal emission follows the optical dust obscuration very closely. NGC 6240 is thus an active interacting remnant viewed at the point of nuclei merging where two AGNs are visible. The results are well consistent with a major merger scenario with the transition from disk galaxies to a spheroid.

\subsection{IRAS 12071-0444: a CT type 2 QSO}

Mid-IR (data from IRS/Spitzer) and X-ray spectroscopy (data from BeppoSAX, Chandra, Suzaku) allow to investigate the nature and peculiar structure of the nearby $(\mathrm{z}=0.128)$ ULIRG $\left(\mathrm{L}_{I R}=9 \times 10^{45} \mathrm{erg} / \mathrm{s}\right)$ IRAS 12071-0444. It is one of the few ULIRGs optically classified as a type 2 AGN and its mid-IR spectrum (in Fig. 5-right, Nardini et al. 2009) shows the typical features of ongoing SB (traced by PAH emission) plus a buried AGN (traced by a reddened power-law).

However, the marginal emission below 10 kev (Fig. 5left) is consistent with the sole SB component, the typical $\mathrm{Fe} \mathrm{K} \alpha$ line is undetected, and the AGN is only seen above $20 \mathrm{keV}$ where the much narrower Suzaku FOV eventually confirms an early tentative detection by BeppoSAX. The X-ray data are well fitted by an absorbed power-law with $\mathrm{N}_{H}=3.5 \times 10^{2} 4 \mathrm{~cm}^{-2}$ and $\mathrm{L}_{2-10} \sim 7 \times 10^{44} \mathrm{erg} / \mathrm{s}$, i.e. about $10 \%$ of the bolometric luminosity is given by the AGN intrinsic emission. The result proves IRAS 12071-0444 as the closest Comptonthick quasar 2 yet known. Finally, a simple visual comparison with the prototypical CT Seyfert NGC 4945 reveals that the reflection efficiency is about a factor of ten lower than for a standard geometry. A peculiar geometry of the absorber is thus required to explain the X-ray/IR data. There are basically two possibilities: (i) a geometrically thick torus, with a small opening angle (i.e. large covering factor). In this case both the direct and reflected components are absorbed. 

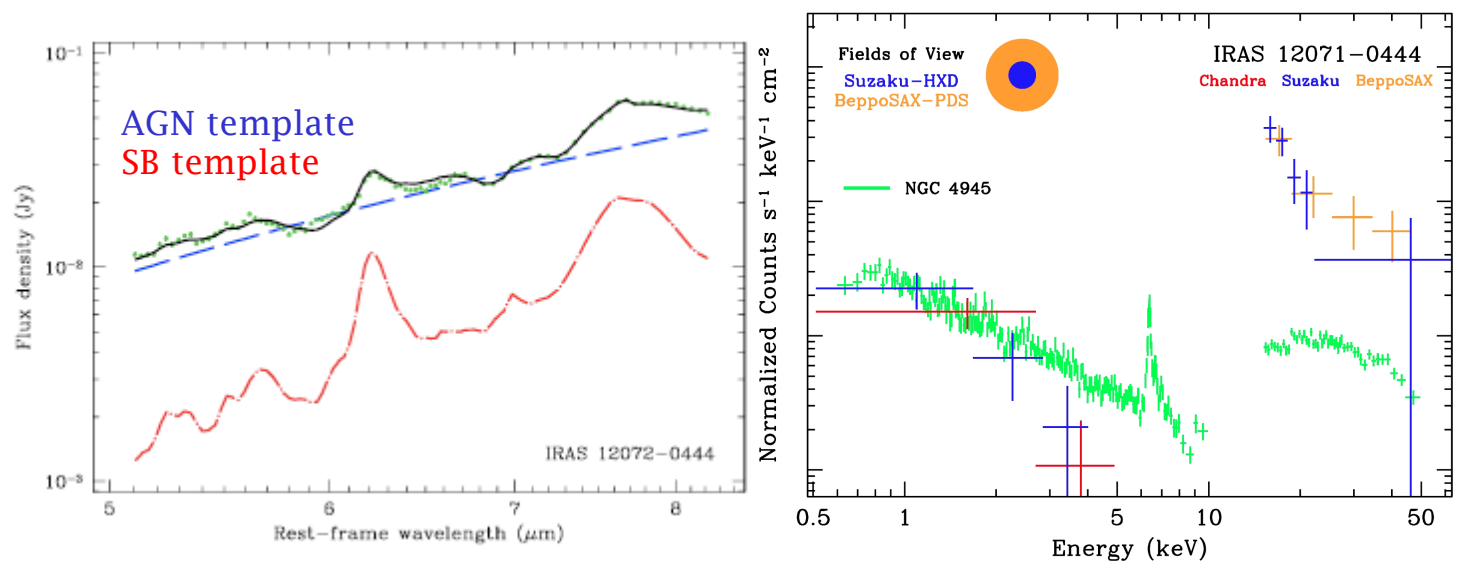

Figure 3: IRAS12071-0444 data. Left: the Spitzer/IRS $\sim 5-8 \mu \mathrm{m}$ spectrum (green dots) is reproduced (black line) by means of an AGN (blue dashed line) and SB (red dot-dashed line) templates. Right: Suzaku data (blue) well agrees with the Chandra/ACIS spectrum (red) in the $0.5-5 \mathrm{keV}$ range, and beyond $15 \mathrm{keV}$ with the BeppoSAX/PDS points (orange). The shaded blue and orange areas in the upper left corner allow to compare Suzaku and BeppoSAX/PDS fields of view respectively. I also compare IRAS 12071-0444 with the NGC 4945 X-ray spectrum observed by XMM-Newton (green). The NGC 4945 data are scaled to match IRAS 12071-0444 at $0.5-10 \mathrm{keV}$.

Alternatively, (ii) the reflection component is absent due to a small compact cloud along the line of sight (i.e. with a small covering factor). The last picture implies both photoelectric Compton absorption without scattering. The resulting intrinsic luminosity exceedes the bolometric one a factor of 3 thus the compact cloud scheme is unphysical. As a probe of the IRAS 120710444 represents a key evolutionary step of the galaxy$\mathrm{BH}$ growth.

\section{Statistical Analysis of Composite Sources}

By selecting large samples of composite SB+AGN sources, it is possible to link the AGN-SF connection processes with the system properties. In the following I show recent results concerning IR and X-ray statistical analysis.

I) The availability of a reliable measure of the relative AGN/SB contribution to ULIRGs (Risaliti et al. 2006, Sani et al. 2008, Nardini et al. 2008) allows a quantitative investigation of the relation between total luminosity and AGN contribution. It is known from optical spectroscopy that the fraction of Seyfert-like systems among IR galaxies grows along with luminosity (Veilleux et al. 1999; Goto 2005). In Nardini et al. 2010 we have quantified how SF and nuclear activity are the primary engine at the opposite ends of the ULIRG luminosity range. The $\mathrm{SB}$ component dominates at $\log \left(L_{I R} / L_{\odot}\right)<12.5$, where the AGN is a significant contributor in only $\sim 30 \%$ of the composite sources. The power supplied by BH accretion grows stronger along the luminosity scale, and ultimately it represents the trigger of the extreme IR activity.

II) The same technique has been applied by Sani et al. 2010 to a sample of local Seyfert galaxies to relate their SB activity with the BH growing parameters. We found that SF activity around Narrow Line Seyfert 1 (NLS1s), i.e. the more accreting AGNs, is larger than around BLS1 of the same AGN luminosity. This result seems to hold over the entire range of distance and luminosity. Moreover, the star formation rate is higher in low black hole mass $\left(\log \left(M_{B H} / M_{\odot}\right)<7\right)$ and high Eddington ratios $\left(\mathrm{L} / \mathrm{L}_{E d d}>0.1\right)$ systems indicating that black hole growth and star formation are occurring simultaneously.

III) With IR and X-ray analysis we can also investigate the SF-AGN connection as a function of obscuration. On one side we do have evidence (Veilleux et al. 2009, Nardini et al. 2010) that, as expected, the fraction of SB-dominated objects shows a constant decline as the radiation field grows harder with absorption features anti-correlating with the optical classification (Siroki et al. 2008). Concerning the individual classes, it is worth noting that a sizable number of very powerful AGN actually lies among LINERs (Low Ionization Narrow Emitting Regions, see also Risaliti, Imanishi \& Sani 2010). Interestingly Seyfert 2, LINERs and HII regions harbor a similar AGN content, supporting the idea of a connection between the SF activity and nuclear obscuration. Moreover, SB dominated galaxies host the most obscured AGNs (see also Georgakakis et al. 2004, Sani et al. 2008, Nardini \& Risaliti 2011). On the other hand, no enhancement of SF is observed 
in obscured AGNs (Rovilos et al. 2012) and the specific SF rate of type 2 AGN hosts is consistent with the value for normal galaxies (Daddi et al. 2007, Sarria et al. 2010, Mainieri et al. 2011). This is thus a debated matter, but we can not relate the SF physics to the $\mathrm{BH}$ physics in a complete and unbiased way because we can not measure e.g. BH mass and accretion rate in type 2 objects.

\section{Catching Feedback in Action}

As mentioned at the beginning of this review, a key ingredient of the merger scenario is feedback. So far a direct detection of the AGN winds able to quench SF in the host galaxy is lacking. Indeed according to Veilleux et al. 2005, $\sim 30 \%$ of QSO show fast winds $(100 \mathrm{~km} / \mathrm{s}$ $\left.<\mathrm{v}_{\text {out }}<1000 \mathrm{~km} / \mathrm{s}\right)$ but the outflow affects the circumnuclear environment only on the broad/narrow line region scales and thus can not inhibit the SF in the host (Muller-Sanchez et al. 2011). Only in nearby sources like Mrk 231 is possible to detect strong molecular outflows (Feruglio et al. 2010, Fisher et al. 2010) acting on kpc scales as expected by QSO-feedback models. The current challenge is to detect such molecular winds at the epoch of the AGN activity peak (i.e. at about redshift 2).

\section{What We Know and What Is Missing}

- While the AGN accretion history is well traced, we still do not know how the SMBH mass function evolves. - If the SF-AGN structure on large scales is well studied, we still lack in constrainig the physical processes on small scales (i.e. with extreme adaptive optics corrections for imaging and IFU observations).

- We are confident that SMBH growth happens simultaneously to the bulk of SF in unobscured composite sources, but the issue for type 2 objects is open. To solve it, we need to identify the entire population and to measure their $\mathrm{M}_{B H}, \mathrm{~L} / \mathrm{L}_{E d d}$, SFR etc.

- It is well known that AGN powers strong winds in local galaxies. Nonetheless most of them affect only the ionized gas on circumnuclear spatial scales and further observational efforts are required to identify $\mathrm{kpc}-$ scale molecular outflows at the peak of AGN activity $(\mathrm{z} \sim 2)$.

\section{Acknowledgement}

I would like Prof. Giovannelli and the SOC for organizing this stimulating workshop.

\section{References}

[1] Barnes, J. E., \& Hernquist, L. E.: 1991, ApJL 370, L65.

[2] Bush, S. J., Wang, Z., Karovska, M., \& Fazio, G. G.: 2008, ApJ 688, 875.

[3] Daddi, E., Alexander, D. M., Dickinson, M., et al.: 2007, ApJ 670, 173.

[4] Di Matteo, T., Springel, V., \& Hernquist, L.: 2005, Nature 433, 604 .

[5] Fabian, A. C.: 1999, MNRAS 308, L39.

[6] Ferrarese, L., \& Merritt, D.: 2000, ApJL, 539, L9. doi: $10.1086 / 312838$

[7] Feruglio, C., Maiolino, R., Piconcelli, E., et al.: 2010, A\&A 518, L155.

[8] Feruglio, C., Fiore, F., Maiolino, R., et al.: 2013, A\&A 549, A51.

[9] Fischer, J., Sturm, E., González-Alfonso, E., et al.: 2010, A\&A 518, L41.

[10] Gebhardt, K., Bender, R., Bower, G., et al.: 2000, ApJL 539, L13. doi:10.1086/312840

[11] Georgakakis, A., Georgantopoulos, I., Vallbé, M., et al.: 2004, MNRAS 349, 135. doi:10.1111/j.1365-2966.2004.07465.x

[12] Goto, T.: 2005, MNRAS 360, 322. doi:10.1111/j.1365-2966.2005.09036.x

[13] Granato, G. L., Silva, L., Monaco, P., et al.: 2001, MNRAS, 324, 757.

[14] Gültekin, K., Richstone, D. O., Gebhardt, K., et al.: 2009, ApJ 698, 198. doi:10.1088/0004-637X/698/1/198

[15] Häring, N., \& Rix, H.-W.: 2004, ApJL 604, L89. doi:10.1086/383567

[16] Hopkins, P. F., Hernquist, L., Cox, T. J., \& Kereš, D.: 2008, ApJS 175, 356. doi:10.1086/524362

[17] Hu J.: 2009, arXiv:0908.2028.

[18] Komossa, S., Burwitz, V., Hasinger, G., et al.: 2003, ApJL 582, L15. doi:10.1086/346145

[19] Kormendy, J., \& Richstone, D.: 1995, ARA\&A 33, 581.

[20] Kormendy, J., Bender, R., \& Cornell, M. E.: 2011, Nature 469, 374. doi:10.1038/nature09694 
[21] Magorrian, J., Tremaine, S., Richstone, D., et al.: 1998, AJ 115, 2285.

[22] Mainieri, V., Bongiorno, A., Merloni, A., et al.: 2011, A\&A 535, A80.

[23] Marconi, A., \& Hunt, L. K.: 2003, ApJL 589, L21.

[24] Marconi, A., Risaliti, G., Gilli, R., et al.: 2004, MNRAS 351, 169.

[25] Medling A. M., Ammons S. M., Max C. E., Davies R. I., Engel H., Canalizo G.: 2011, ApJ 743, 32. doi:10.1088/0004-637X/743/1/32

[26] Menci, N., Fiore, F., Puccetti, S., \& Cavaliere, A.: 2008, ApJ 686, 219.

[27] Merloni, A., \& Heinz, S.: 2008, MNRAS 388, 1011.

[28] Müller-Sánchez, F., Prieto, M. A., Hicks, E. K. S., et al.: 2011, ApJ 739, 69.

[29] Nardini, E., Risaliti, G., Salvati, M., et al.: 2008, MNRAS 385, L130.

[30] Nardini, E., Risaliti, G., Salvati, M., et al.: 2009, MNRAS 399, 1373.

[31] Nardini, E., Risaliti, G., Watabe, Y., Salvati, M., \& Sani, E.: 2010, MNRAS 405, 2505.

[32] Nardini, E., \& Risaliti, G.: 2011, MNRAS 417, 2571 .

[33] Nowak, N., Thomas, J.,Erwin, P., et al.: 2010, MNRAS 403, 646.

[34] Polletta, M., Tajer, M., Maraschi, L., et al.: 2007, ApJ 663, 81. doi:10.1086/518113

[35] Risaliti, G., Sani, E., Maiolino, R., et al.: 2006, ApJL 637, L17. doi:10.1086/500588

[36] Risaliti, G., Maiolino, R., Marconi, A., et al.: 2006, MNRAS 365, 303.

[37] Risaliti, G., Imanishi, M., \& Sani, E.: 2010, MANRAS 401, 197.

[38] Rovilos, E., Comastri, A., Gilli, R., et al.: 2012, A\&A 546, A58.

[39] Sanders, D. B., Soifer, B. T., Elias, J. H., et al. 1988, ApJ 325, 74.
[40] Sani, E., Risaliti, G., Salvati, M., et al.: 2008, ApJ 675, 96. doi:10.1086/523784

[41] Sani, E., Lutz, D., Risaliti, G., et al.: 2010, MNRAS 403, 1246 .

[42] Sani, E., Marconi, A., Hunt, L. K., \& Risaliti, G.: 2011, MNRAS 413, 1479.

[43] Sarria, J. E., Maiolino, R., La Franca, F., et al.: 2010, A\&A 522, L3.

[44] Silk, J., \& Rees, M. J.: 1998, A\&A 331, L1.

[45] Siroki, M. M., Levenson, N. A., Elitzur, M., et al.: 2008, ApJ 678, 729

[46] Soltan, A.: 1982, MNRAS 200, 115.

[47] Tacconi, L. J., Genzel, R., Neri, R., et al.: 2010, Nature 463, 781. doi:10.1038/nature08773

[48] Tacconi, L. J., Neri, R., Genzel, R., et al.: 2013, ApJ 768, 74. doi:10.1088/0004-637X/768/1/74

[49] Veilleux, S., Kim, D.-C., \& Sanders, D. B.: 1999, ApJ 522, 113.

[50] Veilleux, S., Cecil, G., \& Bland-Hawthorn, J.: 2005, ARA\&A 43, 769.

[51] Veilleux, S., Kim, D.-C., Rupke, D. S. N., et al.: 2009, ApJ 701, 587.

\section{DISCUSSION}

CASIANA MUNOZ-TUNON: More obscured AGNs have larger SF, and you mention that no correlation is found between SF and obscuration. What sort of connection do you expect?

ELEONORA SANI: On the basis of hydrodinamical symulations of major mergers, we do expect the co-presence of a violent SB event and a buried AGN at the time of galaxies coalescence. Thus a correlation between the SFR and the AGN obscuration behaviours (e.g. column density) is expected. Analysis based on the COSMOS survey don't find such relation. Nonetheless the issue is still a matter of debate since the lack of this correlation could be due to aperture effects. Moreover deeply embeded AGNs are found in local ULIRGs and high-redshift SMGs. 